\title{
REQUIREMENT OF PROTEOLYTIC CLEAVAGE OF THE MURINE CORONAVIRUS MHV-2 SPIKE PROTEIN FOR FUSION ACTIVITY
}

\author{
Yasuko K. Yamada, ${ }^{1}$ Kazuhiro Takimoto, ${ }^{1}$ Mikiko Yabe, ${ }^{1}$ and \\ Fumihiro Taguchi ${ }^{2}$ \\ ${ }^{1}$ National Institute of Infectious Diseases \\ 4-7-1 Gakuen, Musashimurayama \\ Tokyo 208, Japan \\ ${ }^{2}$ National Institute of Neuroscience, NCNP \\ 4-1-1 Ogawahigashi, Kodaira \\ Tokyo 187, Japan
}

\section{ABSTRACT}

The spike (S) protein of a non-fusogenic murine coronavirus, MHV-2, was compared to that of a variant, MHV-2f, with fusion activity. Two amino acids differed between the $S$ proteins of these viruses; one was located in the signal sequence (amino acid 12) and the other in the putative cleavage site (amino acid 757). To determine which one of these amino acid changes is important for the alteration of fusogenicity, chimeric $\mathrm{S}$ proteins between MHV-2 and $-2 \mathrm{f}$ were constructed and expressed in DBT cells by a vaccinia virus expression system. The results revealed that one amino acid change (Ser to Arg) at position 757 is responsible for the acquisition of fusogenicity of the MHV-2f S protein. This change also altered the susceptibility to proteolytic cleavage of the MHV-2 S protein which was originally uncleavable. We concluded that the non-fusogenic activity of MHV2 results from the lack of cleavage of its $\mathrm{S}$ protein.

\section{INTRODUCTION}

The S protein of murine coronavirus (MHV) mediates syncytium formation. Other fusogenic viruses, such as orthomyxoviruses, paramyxoviruses and retroviruses, require proteolytic cleavage of the surface glycoprotein for fusion activity, since the cleavage exposes the fusion protein on the $\mathrm{N}$-terminus of the membrane-anchored subunit of the surface 
glycoprotein (White, 1990). It is still controversial, however, as to whether S protein cleavage of MHV is required for syncytium formation. Examination of amino acid sequences of the S proteins of MHV-JHMV and -A59 mutants and an MHV-A59 variant suggested that cleavage of the $\mathrm{S}$ protein may not be absolutely necessary for syncytium formation, since the S proteins of these mutants were not cleaved, but still had fusion activity (Bos et al., 1995; Gombold et al., 1993; Stauber et al., 1993; Taguchi, 1993).

MHV-2 is the only strain with a non-fusogenic character among various MHV strains. From MHV-2 virus stock, we recently isolated a variant, $M H V-2 f$, which induces syncytium formation like other fusogenic MHV strains. The comparison of amino acid sequences showed that there were two amino acid differences between the $\mathrm{S}$ proteins of these two viruses. The amino acid at position 12 in the signal sequence was Ser in MHV-2 and Cys in MHV-2f. The amino acid sequence of the cleavage site of MHV-2 was HRARS, while that of MHV-2f was HRARR showing one amino acid change at position 757. Cleavability of the S protein is also different between MHV-2 and $-2 \mathrm{f}$; the S protein of MHV-2 is not cleaved in infected DBT cells, while the S protein of MHV- $2 \mathrm{f}$ is cleaved (Yamada et al., 1997). In order to determine the amino acid involved in the alteration of fusogenicity and cleavability, we constructed chimeric S proteins in which each amino acid change out of two positions was introduced in the MHV-2 S protein and expressed them in DBT cells by a vaccinia virus expression system.

\section{MATERIALS AND METHODS}

\subsection{Construction of Chimeric S Proteins}

The full length coding regions of the S proteins of MHV-2 and $-2 \mathrm{f}$ amplified by RTPCR from infected DBT cells using primers around the initiation and the stop codons (2SPH and 2S-NB, respectively) (Yamada et al., 1997) were cloned into pGEM4Z (Promega) and designated as pS-2 and pS-2f, respectively. To introduce single mutation at nucleotide 35 in the MHV-2 S protein (GenBank Database Accession No. U72635), the S1 subunit of MHV-2f, containing the MHV-2 type cleavage site was amplified from $\mathrm{pS}-2 \mathrm{f}$ using $2 \mathrm{~S}-\mathrm{PH}$ and $2 \mathrm{~S}-2283 \mathrm{~N}$ (antisense nucleotide 2264-2283 of MHV-2) primers. The S2 subunit of MHV-2 was amplified from pS-2 using 2S-2264P (nucleotide 2264 to 2283 of MHV-2) and $2 \mathrm{~S}-\mathrm{NB}$ primers. The full length coding region was then amplified from the mixture of these subunits using $2 \mathrm{~S}-\mathrm{PH}$ and $2 \mathrm{~S}-\mathrm{NB}$ primers, and cloned into pGEM4Z (pS-2f/2). To make the MHV-2 S protein with a single mutation at nucleotide 2271, the S1 subunit of the MHV-2 containing the MHV-2f type cleavage site amplified from pS-2 using $2 \mathrm{~S}-\mathrm{PH}$ and $2 \mathrm{fS}-2283 \mathrm{~N}$ (antisense nucleotide 2264-2283 of MHV-2f) primers, and the S2 subunit of MHV-2f amplified from pS-2f using 2fS-2264P (nucleotide 2264-2283 of MHV-2f) and $2 \mathrm{~S}-\mathrm{NB}$ primers were mixed, amplified using $2 \mathrm{~S}-\mathrm{PH}$ and $2 \mathrm{~S}-\mathrm{NB}$ primers, and cloned into pGEM4Z (pS-2/2f). The nucleotide sequences of pS-2, $-2 \mathrm{f},-2 \mathrm{f} / 2$, and $-2 / 2 \mathrm{f}$ were analyzed by the dideoxy termination labeling method according to the manufacture's instructions (Applied Biosystems).

\subsection{Expression of Chimeric S Proteins}

DBT cells were infected with a recombinant vaccinia virus encoding bacteriophage T7 RNA polymerase (vTF7-3, kindly provided by Dr. B. H. Moss) and then transfected with plasmids, $p S-2,-2 f,-2 f / 2$, and $-2 / 2 f$ with DOTAP transfection reagent (Boehringer 
Mannneim). At $16 \mathrm{hr}$ posttransfection, cytopathic effects were determined. After fixation with chilled acetone, expression of the $\mathrm{S}$ protein was determined by indirect immunofluorescent staining. In other series, transfected cells were washed with PBS and lysed with PBS containing $1 \%$ Nonidet-P40. The lysed samples were centrifuged at $15,000 \mathrm{rpm}$ for $10 \mathrm{~min}$. The supernatants were collected and stored at $-20^{\circ} \mathrm{C}$.

\subsection{Western Blot Analysis}

The lysates were boiled in a sample buffer and electrophoresed in a $7.5 \%$ SDSpolyacrylamide gel. Western blotting analysis was carried out as described previously (Yamada et al., 1997) using an S2 subunit-specific monoclonal antibody (kindly provided by Dr. S. Siddell) and horseradish peroxidase-conjugated anti-mouse IgGs (TAGO). The bands were visualized with PBS containing $0.05 \%$ diaminobenzidine and $0.015 \%$ hydrogen peroxide.

\section{RESULTS}

We previously demonstrated two amino acid differences between fusion-negative MHV-2 and its fusion-positive variant, MHV-2f. One change at position 12 is located in the signal sequence and the other at position 757 is in the putative cleavage site. In order to determine which amino acid change was responsible for the alteration of fusogenicity, chimeric $\mathrm{S}$ proteins were constructed (Figure 1) and cloned into a pGEM vector. Compared with the MHV-2 S protein, $\mathrm{pS}-2 \mathrm{f} / 2$ contained a single amino acid change at position 12 (Ser to Cys), while pS-2/2f had a change at position 757 (Ser to Arg). These chimeras along with the cloned MHV-2 and - $2 \mathrm{f}$ S proteins were transiently expressed in DBT cells by a vaccinia virus expression system. At $16 \mathrm{hr}$ posttransfection, syncytium formation was observed in cells transfected with $\mathrm{pS}-2 \mathrm{f}$ and with $\mathrm{pS}-2 / 2 \mathrm{f}$. On the other hand, syncytium formation was detected in neither cells transfected with pS-2 nor in those transfected with $\mathrm{pS}-2 \mathrm{f} / 2$, even though the expression of the $\mathrm{S}$ protein was detectable by immunofluorescent staining with an $\mathrm{S}$ protein specific monoclonal antibody (Figure 2). These results suggested that one amino acid change at position 757 was responsible for the alteration of the fusogenicity. Although some other amino acid changes were introduced during the process of cloning and constructing chimeras (shown in parentheses in Figure 1), these changes did not seem to affect the fusogenicity.

Since the amino acid at position 757 is located in the putative cleavage site, this mutation could influence the cleavage of the S protein. We analyzed the cleavability of chimeric S proteins by Western blotting with S2-subunit specific monoclonal antibody (Figure 3). Two bands at 150 and $180 \mathrm{kDa}$ were detected in $\mathrm{pS}-2-$ and $-2 \mathrm{f} / 2$-transfected cells, while the 150- and 90-kDa bands were detected in cells transfected with pS-2f and $2 / 2 \mathrm{f}$. The $150-\mathrm{kDa}$ protein may represent a nonglycosylated form of the mature $180-\mathrm{kDa}$

Figure 1. Schematic representation of chimeric $S$ proteins. Open and shaded columns show parts of the $\mathrm{S}$ protein derived from MHV-2 and $-2 \mathrm{f}$, respectively.

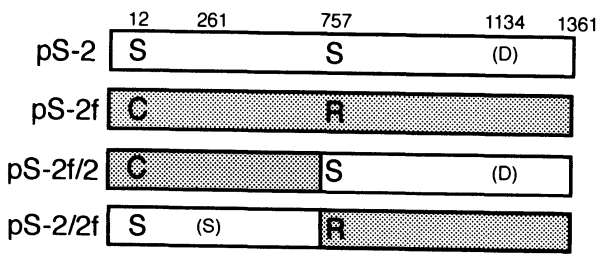



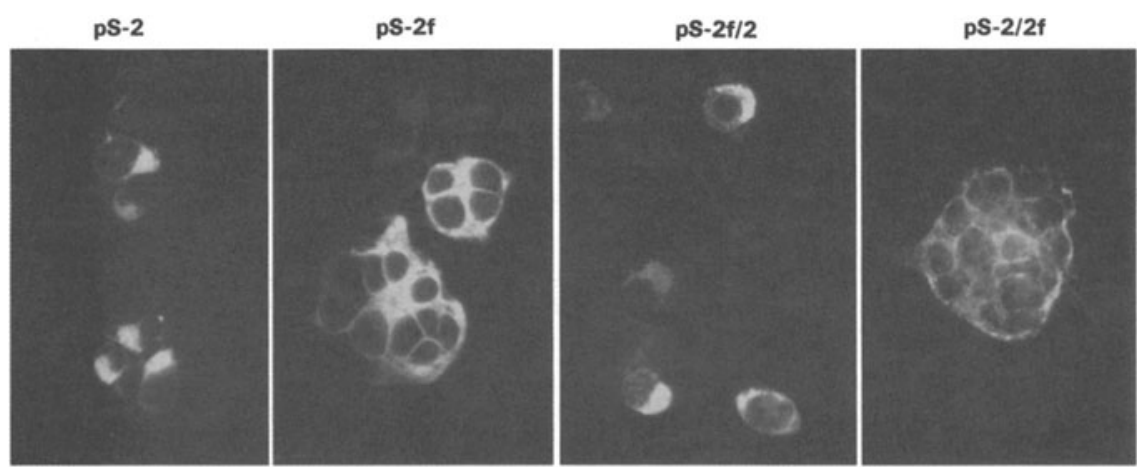

Figure 2. Immunofluorescent staining of DBT cells. Cloned and chimeric $\mathrm{S}$ proteins were expressed by a vaccinia virus expression system. The S protein was detected with anti-S protein specific monoclonal antibody and FITCconjugated anti-mouse IgGs (TAGO) after fixation with chilled acetone.

protein. The mature $180-\mathrm{kDa}$ protein may be cleaved into two subunits of which S2 subunit can be detected as the $90-\mathrm{kDa}$ band by its specific antibody. In cells transfected with pS-2 and with pS-2f $/ 2$, faint bands of around $90 \mathrm{kDa}$ were seen; these probably do not represent $S$ protein cleavage products, because similar faint bands were also seen in control DBT cells infected with vTF7-3. These data showed that the S proteins expressed by $\mathrm{pS}-2$ and $-2 \mathrm{f} / 2$ were not cleaved, while those by $\mathrm{pS}-2 \mathrm{f}$ and $-2 / 2 \mathrm{f}$ were cleaved.

These results demonstrated that MHV-2f acquired the fusion activity as a result of one amino acid change which altered the cleavability of the non-fusogenic MHV-2 S protein. We conclude that the non-fusogenic activity of MHV-2 results from the lack of cleavage of its $\mathrm{S}$ protein.

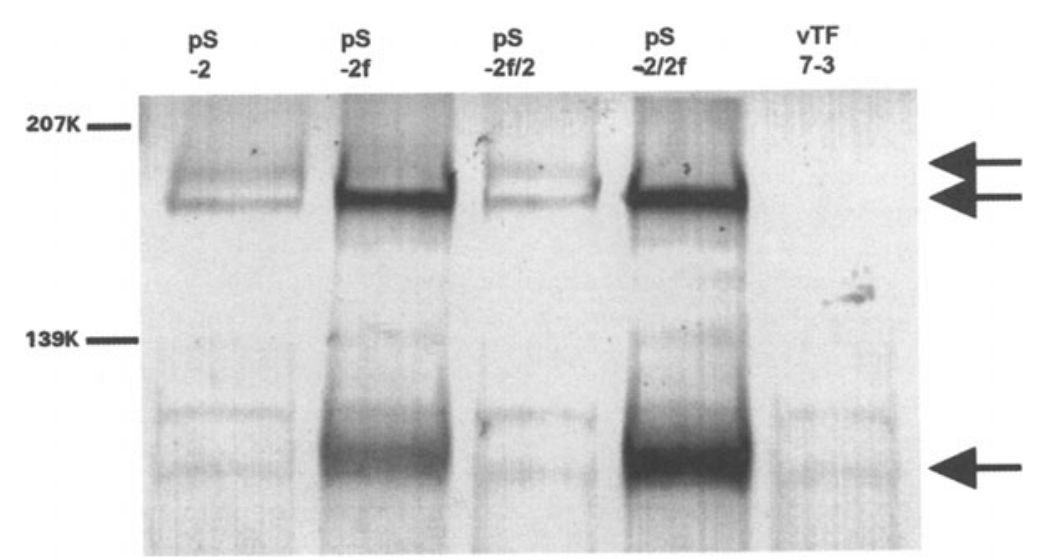

Figure 3. Western blot analysis of S proteins. Cloned and chimeric S proteins were expressed in DBT cells by a vaccinia virus expression system. Cell lysates at $16 \mathrm{hr}$ posttransfection were analyzed as mentioned in Materials and Methods. Arrows indicate from top to bottom 180, 150 and $90 \mathrm{kDa}$. 


\section{DISCUSSION}

Fusogenic MHV strains JHMV and A59, do not absolutely require cleavage of the S protein for their fusion activity, because the $\mathrm{S}$ proteins of these viruses with mutations in the putative cleavage site can induce syncytium, yet are not cleaved (Bos et al., 1995; Gombold et al., 1993; Stauber et al., 1993; Taguchi, 1993). On the other hand, MHV-2 requires cleavage of the $\mathrm{S}$ protein to acquire fusogenicity. Cleavage of the MHV-2 S protein may alter its conformation thereby activating the otherwise non-fusogenic character of this protein. Such a process would be very similar to that of other fusogenic orthomyxo-, paramyxo- and retroviruses (White, 1990). In these viruses, the newly appearing hydrophobic N-terminal region of the membrane-anchored subunit is postulated to work as a fusion peptide. In the N-terminus of the MHV-2 S2 subunit, however, a similar fusion peptide with a stretch of apolar amino acids was not found. We speculated that the region affecting fusion activity is possibly located inside S2, as is postulated for SFV, Sindbis and RSV viruses (White, 1990), because fusion activity is thought not to reside in the $\mathrm{N}$-terminus of the MHV S protein (Keck et al., 1988). The finding that the uncleaved form of the MHV-2 S protein is inactive with respect to fusion is inconsistent with the previously reported result that the S proteins of A59 and JHMV are actively fusogenic without cleavage. Precise examination of the amino acid difference between MHV-2 and other MHV strains would be helpful in defining the molecular basis for the mechanisms of fusion induction which might be different among them.

\section{REFERENCES}

Bos, E. C. W., Heijnen, L., Luytjes, W., and Spaan, W. J. M., 1995, Mutation analysis of the murine coronavirus spike protein: Effect on cell-to-cell fusion, Virology 214: 453-463.

Gombold, J. L., Hingley, S. T., and Weiss, S. R., 1993, Fusion-defective mutants of mouse hepatitis virus A59 contain a mutation in the spike protein cleavage signal, $J$. Virol. 67: 4504-4512.

Keck, J. G., Soe, L. H., Makino, S., Stohlman, S. A., and Lai, M. M. C., 1988, RNA recombination of murine coronaviruses: Recombination between fusion-positive mouse hepatitis A59 and fusion-negative mouse hepatitis virus 2, J. Virol. 62: 1989-1998.

Stauber, R., Pfleiderera, M., and Siddell, S., 1993, Proteolytic cleavage of the murine coronavirus surface glycoprotein is not required for fusion activity, J. Gen. Virol. 74: 183-191.

Taguchi, F., 1993, Fusion formation by the uncleaved spike protein of murine coronavirus JHMV variant cl-2, $J$. Virol. 67: 1195-1202.

White, J. M., 1990, Viral and cellular membrane fusion proteins, Annu. Rev. Physiol. 52: 675-697.

Yamada, Y. K., Takimoto, K., Yabe, M., and Taguchi, F., 1997, Acquired fusion activity of a Murine Coronavirus MHV-2 variant with mutations in the proteolytic cleavage site and the signal sequence of the $\mathrm{S}$ protein, $\mathrm{V}$ rology 227: 215-219. 